International Mathematical Forum, 2, 2007, no. 10, 475 - 498

\title{
Relative Equilibria of a Gyrostat in the Three Body Problem
}

\author{
J. A. Vera and A. Vigueras \\ Departmento de Matemática Aplicada y Estadística \\ Universidad Politécnica de Cartagena \\ 30203 Cartagena (Murcia), Spain \\ juanantonio.vera@upct.es
}

\begin{abstract}
We consider the non-canonical Hamiltonian dynamics of a gyrostat in the three body problem. By means of geometric-mechanics methods we will study the approximate dynamics that arises when we develop the potential in series of Legendre and truncate this in an arbitrary order. Working in the reduced problem, we will study the existence of relative equilibria that we will denominate of Euler and Lagrange in analogy with classic results on the topic. In this way, we generalize the classical results on equilibria of the three-body problem and many of those obtained by other authors using more classic techniques for the case of rigid bodies.
\end{abstract}

Mathematics Subject Classification: 34J15, 34J20, 53D17, 70F07, 70K42, 70H14

Keywords: 3-body problem, gyrostat, relative equilibria, Eulerian, Lagrangian, stability

\section{Introduction}

In the study of configurations of relative equilibria by differential geometry methods or by more classical ones; we will mention here the papers of Wang et al.[10], about the problem of a rigid body in a central Newtonian field; Maciejewski [3], about the problem of two rigid bodies in mutual Newtonian attraction. These papers have been generalized to the case of a gyrostat in a central Newtonian field by Wang et al. [11] and by Mondéjar et al. [4] to the case of two gyrostats in mutual Newtonian attraction. 
For the problem of three rigid bodies we would like to mention that Vidiakin [9] and Duboshine [1] proved the existence of Euler and Lagrange configurations of equilibria when the bodies possess symmetries; Zhuralev et al. [12] made a review of the results up to 1990; Fanny et al. [2] studied the configuration of the equilibria in terms of global variables in the unreduced problem, where the two bodies have spherical distribution of mass and the third is rigid, and in (Mondéjar et al.,[5]), the problem of three bodies where two bodies have spherical distribution of mass and the third one of them is a gyrostat is considered. Working in the reduced problem global considerations about the conditions for relative equilibria are made. Finally, in an approximated model of the dynamics (zero order approximation dynamics), a study of the relative equilibria is made.

In Vera [6] and a series of recent papers (Vera and Vigueras, [7], [8]) we study the non-canonical Hamiltonian dynamics of $n+1$ bodies in Newtonian attraction, where $n$ of them are rigid bodies with spherical distribution of mass or material points and the other one is a triaxial gyrostat.

In this paper, we take $n=2$ and as a first approach to the qualitative study of this system, we will describe the approximate dynamics that arises in a natural way when we take the Legendre development of the potential function and truncate this until an arbitrary order. We will see global conditions on the existence of relative equilibria and in analogy with classic results on the topic, we will study the existence of relative equilibria that we will denominate of Euler and Lagrange type in the case in which $S_{1}, S_{2}$ are spherical or punctual bodies and $S_{0}$ is a gyrostat. We will obtain necessary and sufficient conditions for their existence and we will give explicit expressions of this relative equilibria, useful for the later study of the stability of the same ones.

\section{Equations of the motion}

Following the line of [8] let $S_{0}$ be a gyrostat of mass $m_{0}$ and $S_{1}, S_{2}$ two spherical rigid bodies of masses $m_{1}$ and $m_{2}$. We use the following notation

$$
\begin{array}{ll}
M_{2}=m_{1}+m_{2}, & M_{1}=m_{1}+m_{2}+m_{0}, \\
g_{1}=\frac{m_{1} m_{2}}{M_{2}}, & g_{2}=\frac{m_{0} M_{2}}{M_{1}}
\end{array}
$$

For $\mathbf{u}, \mathbf{v} \in \mathbb{R}^{3}, \mathbf{u} \cdot \mathbf{v}$ is the dot product, $|\mathbf{u}|$ is the Euclidean norm of the vector $\mathbf{u}$ and $\mathbf{u} \times \mathbf{v}$ is the cross product. $\mathbf{I}_{\mathbb{R}^{3}}$ is the identity matrix and $\mathbf{0}$ is the zero matrix of order three. We consider $\mathbb{I}=\operatorname{diag}(A, A, C)$ the diagonal tensor of inertia of the gyrostat. The vector $\mathbf{z}=\left(\Pi, \boldsymbol{\lambda}, \mathbf{p}_{\boldsymbol{\lambda}}, \boldsymbol{\mu}, \mathbf{p}_{\mu}\right) \in \mathbb{R}^{15}$ is a generic element of the twice reduced problem obtained using the symmetries of the system. The vector $\Pi=\mathbb{I} \Omega+\mathbf{l}_{r}$ is the total rotational angular momentum of 
the gyrostat in the body frame, which is attached to its rigid part and whose axes have the direction of the principal axes of inertia of $S_{0}$ and $\mathbf{l}_{r}=(0,0, l)$ is the constant gyrostatic momentum. The elements $\boldsymbol{\lambda}, \boldsymbol{\mu}, \mathbf{p}_{\boldsymbol{\lambda}}$ and $\mathbf{p}_{\mu}$ are respectively the barycentric coordinates and the linear momenta expressed in the body frame $\mathfrak{J}$.

The twice reduced Hamiltonian of the system, obtained by the action of the group $\mathbf{S E}(3)$, has the following expression

$$
\mathcal{H}(\mathbf{z})=\frac{\left|\mathbf{p}_{\boldsymbol{\lambda}}\right|^{2}}{2 g_{1}}+\frac{\left|\mathbf{p}_{\boldsymbol{\mu}}\right|^{2}}{2 g_{2}}+\frac{1}{2} \Pi \mathbb{I}^{-1} \Pi-\mathbf{l}_{r} \cdot \mathbb{I}^{-1} \Pi+\mathcal{V}(\boldsymbol{\lambda}, \boldsymbol{\mu})
$$

Let $\mathbf{M}=\mathbb{R}^{15}$, and we consider the manifold $(M,\{\},, \mathcal{H})$, with Poisson brackets $\{$,$\} defined by means of the Poisson tensor$

$$
\mathbf{B}(\mathbf{z})=\left(\begin{array}{ccccc}
\widehat{\Pi} & \widehat{\boldsymbol{\lambda}} & \widehat{\mathbf{p}_{\boldsymbol{\lambda}}} & \widehat{\boldsymbol{\mu}} & \widehat{\mathbf{p}_{\mu}} \\
\widehat{\boldsymbol{\lambda}} & \mathbf{0} & \mathbf{I}_{\mathbb{R}^{3}} & \mathbf{0} & \mathbf{0} \\
\widehat{\mathbf{p}_{\boldsymbol{\lambda}}} & -\mathbf{I}_{\mathbb{R}^{3}} & \mathbf{0} & \mathbf{0} & \mathbf{0} \\
\widehat{\boldsymbol{\mu}} & \mathbf{0} & \mathbf{0} & \mathbf{0} & \mathbf{I}_{\mathbb{R}^{3}} \\
\widehat{\mathbf{p}_{\mu}} & \mathbf{0} & \mathbf{0} & -\mathbf{I}_{\mathbb{R}^{3}} & \mathbf{0}
\end{array}\right)
$$

In $\mathbf{B}(\mathbf{z}), \widehat{\mathbf{v}}$ is considered to be the image of the vector $\mathbf{v} \in \mathbb{R}^{3}$ by the standard isomorphism between the Lie Algebras $\mathbb{R}^{3}$ and $\mathfrak{s o}(3)$, i.e.

$$
\widehat{\mathbf{v}}=\left(\begin{array}{ccc}
0 & -v_{3} & v_{2} \\
v_{3} & 0 & -v_{1} \\
-v_{2} & v_{1} & 0
\end{array}\right)
$$

The equations of the motion is given by the following expression

$$
\frac{d \mathbf{z}}{d t}=\{\mathbf{z}, \mathcal{H}(\mathbf{z})\}(\mathbf{z})=\mathbf{B}(\mathbf{z}) \boldsymbol{\nabla}_{\mathbf{z}} \mathcal{H}(\mathbf{z})
$$

with $\boldsymbol{\nabla}_{\mathbf{u}} \mathcal{V}$ is the gradient of $\mathcal{V}$ with respect to an arbitrary vector $\mathbf{u}$.

Developing $\{\mathbf{z}, \mathcal{H}(\mathbf{z})\}$, we obtain the following group of vectorial equations of the motion

$$
\begin{array}{ll}
\frac{d \Pi}{d t}=\Pi \times \Omega+\boldsymbol{\lambda} \times \nabla_{\boldsymbol{\lambda}} \mathcal{V}+\boldsymbol{\mu} \times \nabla_{\boldsymbol{\mu}} \mathcal{V} \\
\frac{d \boldsymbol{\lambda}}{d t}=\frac{\mathbf{p}_{\boldsymbol{\lambda}}}{g_{1}}+\boldsymbol{\lambda} \times \Omega, \quad \frac{d \mathbf{p}_{\boldsymbol{\lambda}}}{d t}=\mathbf{p}_{\boldsymbol{\lambda}} \times \Omega-\nabla_{\boldsymbol{\lambda}} \mathcal{V} \\
\frac{d \boldsymbol{\mu}}{d t}=\frac{\mathbf{p}_{\boldsymbol{\mu}}}{g_{2}}+\boldsymbol{\mu} \times \Omega, & \frac{d \mathbf{p}_{\boldsymbol{\mu}}}{d t}=\mathbf{p}_{\boldsymbol{\mu}} \times \Omega-\nabla_{\boldsymbol{\mu}} \mathcal{V}
\end{array}
$$

We denote by $\mathbf{z}_{e}=\left(\Pi_{e}, \boldsymbol{\lambda}^{e}, \mathbf{p}_{\boldsymbol{\lambda}}^{e}, \boldsymbol{\mu}^{e}, \mathbf{p}_{\mu}^{e}\right)$ a generic relative equilibrium of

$$
\frac{d \mathbf{z}}{d t}=\{\mathbf{z}, \mathcal{H}(\mathbf{z})\}=\mathbf{B}(\mathbf{z}) \boldsymbol{\nabla}_{\mathbf{z}} \mathcal{H}(\mathbf{z})
$$


Besides of relative equilibria type Euler or Lagrange, in which the angular velocity $\Omega_{e}$ of the gyrostat is orthogonal to the straight line or plane of the centers of masses of the bodies, we will study the existence of others in those which $\Omega_{e}$ is in the plane that generate the two vectors $\boldsymbol{\lambda}^{e}$ and $\boldsymbol{\mu}^{e}$. We will give necessary and sufficient conditions for existence of these relative equilibria. This results generalize those obtained by Fanny et al. [2], Vidiakin [9] and Duboshin [1] for a rigid body in the three body problem.

The dynamics $\frac{d \mathbf{z}}{d t}=\mathbf{B}(\mathbf{z}) \boldsymbol{\nabla}_{\mathbf{z}} \mathcal{H}(\mathbf{z})$ has three invariant manifolds $\mathbf{M}_{C}, \mathbf{M}_{B}$, $\mathbf{M}_{A}$ that facilitate the study of certain relative equilibria in a next paper.

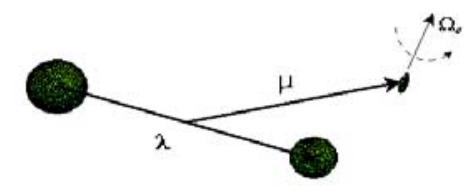

Figure 1: Gyrostat in the three body problem

\section{Approximate Hamiltonian dynamics}

To simplify the problem we assume that the gyrostat $S_{0}$ is symmetrical around the third axis of inertia $O z$ and with respect to the plane $O x y$. If the mutual distances are bigger than the individual dimensions of the bodies, then we can develop the potential in quickly convergent series. Under these hypotheses, we will be able to carry out a study of certain relative equilibria in different approximate dynamics.

The potential function is given by the formula,

$$
\mathcal{V}(\boldsymbol{\lambda}, \boldsymbol{\mu})=-\left(\frac{G m_{1} m_{2}}{|\boldsymbol{\lambda}|}+\int_{S_{0}} \frac{G m_{1} d m(\mathbf{Q})}{\left|\mathbf{Q}+\boldsymbol{\mu}+\frac{m_{2}}{M_{2}} \boldsymbol{\lambda}\right|}+\int_{S_{0}} \frac{G m_{2} d m(\mathbf{Q})}{\left|\mathbf{Q}+\boldsymbol{\mu}-\frac{m_{1}}{M_{2}} \boldsymbol{\lambda}\right|}\right)
$$

Applying the Legendre development of the potential, we have

$$
\mathcal{V}(\boldsymbol{\lambda}, \boldsymbol{\mu})=-\left(\frac{G m_{1} m_{2}}{|\boldsymbol{\lambda}|}+\sum_{i=0}^{\infty} \frac{G m_{1} A_{2 i}}{\left|\boldsymbol{\mu}+\frac{m_{2}}{M_{2}} \boldsymbol{\lambda}\right|^{2 i+1}}+\sum_{i=0}^{\infty} \frac{G m_{2} A_{2 i}}{\left|\boldsymbol{\mu}-\frac{m_{1}}{M_{2}} \boldsymbol{\lambda}\right|^{2 i+1}}\right)
$$

where $A_{0}=m_{0}, A_{2}=(C-A) / 2$ and $A_{2 i}$ are certain coefficients related with the geometry of the gyrostat, see Vera et al. [8]. 
Definition 1 We call approximate potential of order $k$, to the following expression

$$
\mathcal{V}^{(k)}(\boldsymbol{\lambda}, \boldsymbol{\mu})=-\left(\frac{G m_{1} m_{2}}{|\boldsymbol{\lambda}|}+\sum_{i=0}^{k} \frac{G m_{1} A_{2 i}}{\left|\boldsymbol{\mu}+\frac{m_{2}}{M_{2}} \boldsymbol{\lambda}\right|^{2 i+1}}+\sum_{i=0}^{k} \frac{G m_{2} A_{2 i}}{\left|\boldsymbol{\mu}-\frac{m_{1}}{M_{2}} \boldsymbol{\lambda}\right|^{2 i+1}}\right)
$$

It is easy to demonstrate the following lemmas.

Lemma 1 Given the approximate potential of order $k$, we have

$$
\begin{gathered}
\nabla_{\boldsymbol{\lambda}} \mathcal{V}^{(k)}=\left(\frac{G m_{1} m_{2} \boldsymbol{\lambda}}{|\boldsymbol{\lambda}|^{3}}+\frac{G m_{1} m_{2}}{M_{2}} \sum_{i=0}^{k} \frac{\left(\boldsymbol{\mu}+\frac{m_{2}}{M_{2}} \boldsymbol{\lambda}\right)(2 i+1) A_{2 i}}{\left|\boldsymbol{\mu}+\frac{m_{2}}{M_{2}} \boldsymbol{\lambda}\right|^{2 i+3}}\right. \\
\left.-\frac{G m_{1} m_{2}}{M_{2}} \sum_{i=0}^{k} \frac{\left(\boldsymbol{\mu}-\frac{m_{1}}{M_{2}} \boldsymbol{\lambda}\right)(2 i+1) A_{2 i}}{\left|\boldsymbol{\mu}-\frac{m_{1}}{M_{2}} \boldsymbol{\lambda}\right|^{2 i+3}}\right) \\
\nabla_{\boldsymbol{\mu}} \mathcal{V}^{(k)}=G m_{1} \sum_{i=0}^{k} \frac{\left(\boldsymbol{\mu}+\frac{m_{2}}{M_{2}} \boldsymbol{\lambda}\right)(2 i+1) A_{2 i}}{\left|\boldsymbol{\mu}+\frac{m_{2}}{M_{2}} \boldsymbol{\lambda}\right|^{2 i+3}}+G m_{2} \sum_{i=0}^{k} \frac{\left(\boldsymbol{\mu}-\frac{m_{1}}{M_{2}} \boldsymbol{\lambda}\right)(2 i+1) A_{2 i}}{\left|\boldsymbol{\mu}-\frac{m_{1}}{M_{2}} \boldsymbol{\lambda}\right|^{2 i+3}}
\end{gathered}
$$

Also, the following identities are verified

$$
\boldsymbol{\nabla}_{\boldsymbol{\lambda}} \mathcal{V}^{(k)}=\widetilde{A}_{11} \boldsymbol{\lambda}+\widetilde{A}_{12} \boldsymbol{\mu}, \quad \boldsymbol{\nabla}_{\boldsymbol{\mu}} \mathcal{V}^{(k)}=\widetilde{A}_{21} \boldsymbol{\lambda}+\widetilde{A}_{22} \boldsymbol{\mu}
$$

being

$$
\begin{gathered}
\widetilde{A}_{11}(\boldsymbol{\lambda}, \boldsymbol{\mu})=\frac{G m_{1} m_{2}}{|\boldsymbol{\lambda}|^{3}}+\frac{G m_{1} m_{2}^{2}}{M_{2}^{2}}\left(\sum_{i=0}^{k} \frac{\alpha_{i}}{\left|\boldsymbol{\mu}+\frac{m_{2}}{M_{2}} \boldsymbol{\lambda}\right|^{2 i+3}}\right) \\
+\frac{G m_{1}^{2} m_{2}}{M_{2}^{2}}\left(\sum_{i=0}^{k} \frac{\alpha_{i}}{\left|\boldsymbol{\mu}-\frac{m_{1}}{M_{2}} \boldsymbol{\lambda}\right|^{2 i+3}}\right) \\
\widetilde{A}_{12}(\boldsymbol{\lambda}, \boldsymbol{\mu})=\frac{G m_{1} m_{2}}{M_{2}}\left(\sum_{i=0}^{k} \frac{\alpha_{i}}{\left|\boldsymbol{\mu}+\frac{m_{2}}{M_{2}} \boldsymbol{\lambda}\right|^{2 i+3}}-\sum_{i=0}^{k} \frac{\alpha_{i}}{\left|\boldsymbol{\mu}-\frac{m_{1}}{M_{2}} \boldsymbol{\lambda}\right|^{2 i+3}}\right) \\
\widetilde{A}_{22}(\boldsymbol{\lambda}, \boldsymbol{\mu})=G m_{1}\left(\sum_{i=0}^{k} \frac{\alpha_{i}}{\left|\boldsymbol{\mu}+\frac{m_{2}}{M_{2}} \boldsymbol{\lambda}\right|^{2 i+3}}\right)+G m_{2}\left(\sum_{i=0}^{k} \frac{\alpha_{i}}{\left|\boldsymbol{\mu}-\frac{m_{1}}{M_{2}} \boldsymbol{\lambda}\right|^{2 i+3}}\right)
\end{gathered}
$$

with coefficients $\alpha_{0}=m_{0}, \alpha_{1}=3 / 2(C-A), \alpha_{i}=(2 i+1) A_{2 i}$ for $i \geqslant 1$. 
Definition 2 Let be $\mathbf{M}=\mathbb{R}^{15}$ and the manifold $\left(M,\{\},, \mathcal{H}_{I I}^{k}\right)$, with Poisson brackets $\{$,$\} defined by means of the Poisson tensor$

$$
\mathbf{B}(\mathbf{z})=\left(\begin{array}{ccccc}
\widehat{\Pi} & \widehat{\boldsymbol{\lambda}} & \widehat{\mathbf{p}_{\boldsymbol{\lambda}}} & \widehat{\boldsymbol{\mu}} & \widehat{\mathbf{p}_{\mu}} \\
\widehat{\boldsymbol{\lambda}_{1}} & \mathbf{0} & \mathbf{I}_{\mathbb{R}^{3}} & \mathbf{0} & \mathbf{0} \\
\widehat{\mathbf{p}_{\boldsymbol{\lambda}}} & -\mathbf{I}_{\mathbb{R}^{3}} & \mathbf{0} & \mathbf{0} & \mathbf{0} \\
\widehat{\boldsymbol{\mu}} & \mathbf{0} & \mathbf{0} & \mathbf{0} & \mathbf{I}_{\mathbb{R}^{3}} \\
\widehat{\mathbf{p}_{\mu}} & \mathbf{0} & \mathbf{0} & -\mathbf{I}_{\mathbb{R}^{3}} & \mathbf{0}
\end{array}\right)
$$

We call approximate dynamics of order $k$ to the differential equations of motion given by the following expression

$$
\frac{d \mathbf{z}}{d t}=\left\{\mathbf{z}, \mathcal{H}_{I I}^{k}(\mathbf{z})\right\}_{I I}(\mathbf{z})=\mathbf{B}(\mathbf{z}) \boldsymbol{\nabla}_{\mathbf{z}} \mathcal{H}_{I I}^{k}(\mathbf{z})
$$

being

$$
\mathcal{H}_{I I}^{k}(\mathbf{z})=\frac{\left|\mathbf{p}_{\boldsymbol{\lambda}}\right|^{2}}{2 g_{1}}+\frac{\left|\mathbf{p}_{\boldsymbol{\mu}}\right|^{2}}{2 g_{2}}+\frac{1}{2} \Pi \mathbb{I}^{-1} \Pi-\mathbf{l}_{r} \cdot \mathbb{I}^{-1} \Pi+\mathcal{V}^{(k)}(\boldsymbol{\lambda}, \boldsymbol{\mu})
$$

On the other hand, it is easy to verify that

$$
\left.\nabla_{\mathbf{z}}\left(|\Pi|^{2}\right)\right) \mathbf{B}(\mathbf{z}) \nabla_{\mathbf{z}} \mathcal{H}_{I I}^{k}(\mathbf{z})=0
$$

and similarly when the gyrostat is of revolution

$$
\nabla_{\mathbf{z}}\left(\boldsymbol{\pi}_{3}\right) \mathbf{B}(\mathbf{z}) \nabla_{\mathbf{z}} \mathcal{H}_{I I}^{k}(\mathbf{z})=0
$$

where $\boldsymbol{\pi}_{3}$ is the third component of the rotational angular momentum of the gyrostat. It is verified the following property.

Proposition 2 In the approximate dynamics of order $k,|\Pi|^{2}$ is an integral of motion and also when the gyrostat is of revolution $\boldsymbol{\pi}_{3}$ is another integral of motion.

\section{Relative Equilibria}

The relative equilibria are the equilibria of the twice reduced problem whose Hamiltonian function is obtained in [8] for the case $n=2$, if we denote by $\mathbf{z}_{e}=$ $\left(\Pi_{e}, \boldsymbol{\lambda}^{e}, \mathbf{p}_{\boldsymbol{\lambda}}^{e}, \boldsymbol{\mu}^{e}, \mathbf{p}_{\mu}^{e}\right)$ a generic relative equilibrium of an approximate dynamics of order $k$, then this verifies the equations

$$
\begin{gathered}
\Pi_{e} \times \Omega_{e}+\boldsymbol{\lambda}^{e} \times\left(\boldsymbol{\nabla}_{\boldsymbol{\lambda}} \mathcal{V}^{(k)}\right)_{e}+\boldsymbol{\mu}^{e} \times\left(\boldsymbol{\nabla}_{\boldsymbol{\mu}} \mathcal{V}^{(k)}\right)_{e}=\mathbf{0} \\
\frac{\mathbf{p}_{\boldsymbol{\lambda}}^{e}}{g_{1}}+\boldsymbol{\lambda}^{e} \times \Omega_{e}=\mathbf{0}, \quad \mathbf{p}_{\boldsymbol{\lambda}}^{e} \times \Omega_{e}=\left(\boldsymbol{\nabla}_{\boldsymbol{\lambda}} \mathcal{V}^{(k)}\right)_{e} \\
\frac{\mathbf{p}_{\boldsymbol{\mu}}^{e}}{g_{2}}+\boldsymbol{\mu}^{e} \times \Omega_{e}=\mathbf{0}, \quad \mathbf{p}_{\boldsymbol{\mu}}^{e} \times \Omega_{e}=\left(\boldsymbol{\nabla}_{\boldsymbol{\mu}} \mathcal{V}^{(k)}\right)_{e} .
\end{gathered}
$$


Also by virtue of the relationships obtained in [8], we have the following result.

Lemma 3 If $\mathbf{z}_{e}=\left(\Pi_{e}, \boldsymbol{\lambda}^{e}, \mathbf{p}_{\boldsymbol{\lambda}}^{e}, \boldsymbol{\mu}^{e}, \mathbf{p}_{\mu}^{e}\right)$ is a relative equilibrium of an approximate dynamics of order $k$ the following relationships are verified

$$
\begin{gathered}
\Pi_{e} \times \Omega_{e}=\mathbf{0} \\
\left|\Omega_{e}\right|^{2}\left|\boldsymbol{\lambda}^{e}\right|^{2}-\left(\boldsymbol{\lambda}^{e} \cdot \Omega_{e}\right)^{2}=\frac{1}{g_{1}}\left(\boldsymbol{\lambda}^{e} \cdot\left(\nabla_{\boldsymbol{\lambda}} \mathcal{V}^{(k)}\right)_{e}\right) \\
\left|\Omega_{e}\right|^{2}\left|\boldsymbol{\mu}^{e}\right|^{2}-\left(\boldsymbol{\mu}^{e} \cdot \Omega_{e}\right)^{2}=\frac{1}{g_{2}}\left(\boldsymbol{\mu}^{e} \cdot\left(\boldsymbol{\nabla}_{\boldsymbol{\mu}} \mathcal{V}^{(k)}\right)_{e}\right)
\end{gathered}
$$

The last two previous identities will be used to obtain necessary conditions for the existence of relative equilibria in this approximate dynamics. From the first previous equation, the following property is deduced.

Proposition 4 In the relative equilibria, for any approximate dynamics of arbitrary order, moments are not exercised on the gyrostat.

We will study certain relative equilibria in the approximate dynamics supposing that the vectors $\Omega_{e}, \boldsymbol{\lambda}^{e}, \boldsymbol{\mu}^{e}$ satisfy special geometric properties.

Definition 3 We say that $\mathbf{z}_{e}$ is a relative equilibrium type Euler in an approximate dynamics of order $k$ when, $\boldsymbol{\lambda}^{e}, \boldsymbol{\mu}^{e}$ are proportional and $\Omega_{e}$ is perpendicular to the straight line that these generate.

Definition 4 We say that $\mathbf{z}_{e}$ is a relative equilibrium of Lagrange type, in an approximate dynamics of order $k$, if $\boldsymbol{\lambda}^{e}, \boldsymbol{\mu}^{e}$ are not proportional and $\Omega_{e}$ is perpendicular to the plane that these generate.

Next we obtain necessary and sufficient conditions for the existence of equilibria of Euler and Lagrange type.

\section{Relative equilibria of Euler type}

According to the relative position of the gyrostat $S_{0}$ with respect to $S_{1}$ and $S_{2}$ there are three possible equilibrium configurations: a) $S_{0} S_{2} S_{1}$, b) $S_{2} S_{0} S_{1}$ and c) $S_{2} S_{1} S_{0}$. 


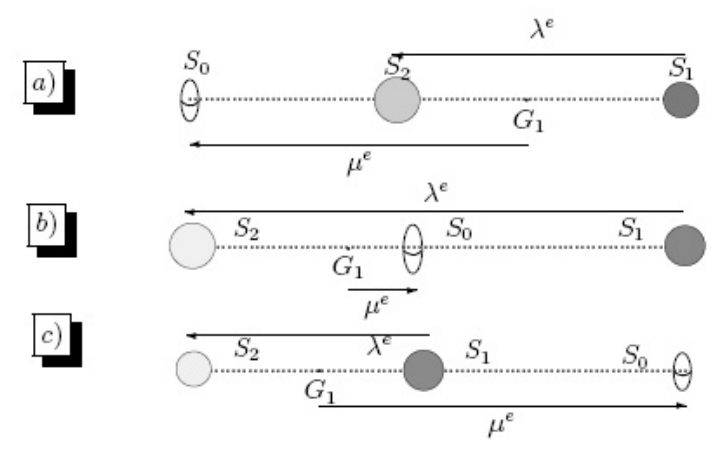

Figure 2: Eulerian configurations

\subsection{Necessary condition of existence}

Lemma 5 If $\mathbf{z}_{e}=\left(\Pi_{e}, \boldsymbol{\lambda}^{e}, \mathbf{p}_{\boldsymbol{\lambda}}^{e}, \boldsymbol{\mu}^{e}, \mathbf{p}_{\mu}^{e}\right)$ is a relative equilibrium of Euler type, then for the configuration $S_{0} S_{2} S_{1}$ we have

$$
\left|\boldsymbol{\mu}^{e}+\frac{m_{1}}{M_{2}} \boldsymbol{\lambda}^{e}\right|=\left|\boldsymbol{\lambda}^{e}\right|+\left|\boldsymbol{\mu}^{e}-\frac{m_{2}}{M_{2}} \boldsymbol{\lambda}^{e}\right| .
$$

In a similar way, for the configuration $S_{2} S_{0} S_{1}$ we have

$$
\left|\boldsymbol{\lambda}^{e}\right|=\left|\boldsymbol{\mu}^{e}-\frac{m_{1}}{M_{2}} \boldsymbol{\lambda}^{e}\right|+\left|\boldsymbol{\mu}^{e}+\frac{m_{2}}{M_{2}} \boldsymbol{\lambda}^{e}\right| .
$$

Finally, for the configuration $S_{2} S_{1} S_{0}$ we have

$$
\left|\boldsymbol{\mu}^{e}-\frac{m_{2}}{M_{2}} \boldsymbol{\lambda}^{e}\right|=\left|\boldsymbol{\mu}^{e}+\frac{m_{1}}{M_{2}} \boldsymbol{\lambda}^{e}\right|+\left|\boldsymbol{\lambda}^{e}\right| .
$$

Next we study necessary and sufficient conditions for the existence of relative equilibria of Euler type for the configuration $S_{0} S_{2} S_{1}$; the other configurations are studied in a similar way. If $\mathbf{z}_{e}$ is a relative equilibrium of Euler type, in the configuration $S_{0} S_{2} S_{1}$ in an approximate dynamics of order $k$, we have

$$
\begin{gathered}
g_{1}\left|\Omega_{e}\right|^{2}\left|\boldsymbol{\lambda}^{e}\right|^{2}=\boldsymbol{\lambda}^{e} \cdot\left(\nabla_{\boldsymbol{\lambda}} \mathcal{V}^{(k)}\right)_{e} \\
g_{2}\left|\Omega_{e}\right|^{2}\left|\boldsymbol{\mu}^{e}\right|^{2}=\boldsymbol{\mu}^{e} \cdot\left(\boldsymbol{\nabla}_{\boldsymbol{\mu}} \mathcal{V}^{(k)}\right)_{e}
\end{gathered}
$$

and

$$
\begin{aligned}
\boldsymbol{\mu}^{e}-\frac{m_{1}}{M_{2}} \boldsymbol{\lambda}^{e} & =\rho \boldsymbol{\lambda}^{e}, \quad \boldsymbol{\mu}^{e}+\frac{m_{2}}{M_{2}} \boldsymbol{\lambda}^{e}=(1+\rho) \boldsymbol{\lambda}^{e} \\
\boldsymbol{\mu}^{e} & =\frac{\left((1+\rho) m_{1}+\rho m_{2}\right)}{M_{2}} \boldsymbol{\lambda}^{e}
\end{aligned}
$$


where $\rho \in(0,+\infty)$ in the case $a), \rho \in(-1,0)$ in the case $b)$ and $\rho \in(-\infty,-1)$ in the case $c$ ). And it is possible to obtain the following expressions

$$
\left(\nabla_{\boldsymbol{\lambda}} \mathcal{V}^{(k)}\right)_{e}=\widetilde{T}_{1}(\rho) \boldsymbol{\lambda}^{e}, \quad\left(\boldsymbol{\nabla}_{\boldsymbol{\mu}} \mathcal{V}^{(k)}\right)_{e}=\widetilde{T}_{2}(\rho) \boldsymbol{\lambda}^{e}
$$

where

$$
\begin{gathered}
\widetilde{T}_{1}(\rho)=\frac{G m_{1} m_{2}}{\left|\boldsymbol{\lambda}^{e}\right|^{3}}+\frac{G m_{1} m_{2}}{M_{2}}\left(\sum_{i=0}^{k} \frac{\alpha_{i}}{\left|\boldsymbol{\lambda}^{e}\right|^{2 i+3}}\left(\frac{1+\rho}{|1+\rho|^{2 i+3}}-\frac{\rho}{|\rho|^{2 i+3}}\right)\right) \\
\widetilde{T}_{2}(\rho)=\sum_{i=0}^{k} \frac{G \alpha_{i}}{\left|\boldsymbol{\lambda}^{e}\right|^{2 i+3}}\left(\frac{m_{1}(1+\rho)}{|1+\rho|^{2 i+3}}+\frac{m_{2} \rho}{|\rho|^{2 i+3}}\right)
\end{gathered}
$$

Restricting us to the case $a$ ) we have

$$
\begin{gathered}
\widetilde{T}_{1}(\rho)=\frac{G m_{1} m_{2}}{\left|\lambda^{e}\right|^{3}}+\frac{G m_{1} m_{2}}{M_{2}}\left(\sum_{i=0}^{k} \frac{\alpha_{i}}{\left|\lambda^{e}\right|^{2 i+3}}\left(\frac{1}{(1+\rho)^{2 i+2}}-\frac{1}{\rho^{2 i+2}}\right)\right) \\
\widetilde{T}_{2}(\rho)=\sum_{i=0}^{k} \frac{G \alpha_{i}}{\left|\lambda^{e}\right|^{2 i+3}}\left(\frac{m_{1}}{(1+\rho)^{2 i+2}}+\frac{m_{2}}{\rho^{2 i+2}}\right) .
\end{gathered}
$$

Now, from the identities

$$
\begin{gathered}
\boldsymbol{\lambda}^{e} \cdot\left(\nabla_{\boldsymbol{\lambda}} \mathcal{V}^{(k)}\right)_{e}=\left|\boldsymbol{\lambda}^{e}\right|^{2} \widetilde{T}_{1}(\rho) \\
\boldsymbol{\mu}^{e} \cdot\left(\boldsymbol{\nabla}_{\boldsymbol{\mu}} \mathcal{V}^{(k)}\right)_{e}=\frac{\left((1+\rho) m_{1}+\rho m_{2}\right)}{M_{2}}\left|\boldsymbol{\lambda}^{e}\right|^{2} \widetilde{T}_{2}(\rho)
\end{gathered}
$$

we deduce the following equations

$$
\begin{gathered}
\left|\Omega_{e}\right|^{2}=\frac{\widetilde{T}_{1}(\rho)}{g_{1}} \\
\left|\Omega_{e}\right|^{2}=\frac{M_{2} \widetilde{T}_{2}(\rho)}{g_{2}\left((1+\rho) m_{1}+\rho m_{2}\right)} .
\end{gathered}
$$

Then for a relative equilibrium of Euler type $\rho$ must be a positive real root of the following equation

$$
g_{2}\left((1+\rho) m_{1}+\rho m_{2}\right) \widetilde{T}_{1}(\rho)=g_{1} M_{2} \widetilde{T}_{2}(\rho)
$$

We summarize all these results in the following proposition. 
Proposition 6 If $\mathbf{z}_{e}=\left(\Pi_{e}, \boldsymbol{\lambda}^{e}, \mathbf{p}_{\boldsymbol{\lambda}}^{e}, \boldsymbol{\mu}^{e}, \mathbf{p}_{\mu}^{e}\right)$ is an Eulerian relative equilibrium in the configuration $S_{0} S_{2} S_{1}$, the equation

$$
g_{2}\left((1+\rho) m_{1}+\rho m_{2}\right) \widetilde{T}_{1}(\rho)=g_{1} M_{2} \widetilde{T}_{2}(\rho)
$$

has, at least, a positive real root; where the functions $\widetilde{T}_{1}(\rho)$ and $\widetilde{T}_{2}(\rho)$ are given by

$$
\begin{gathered}
\widetilde{T}_{1}(\rho)=\frac{G m_{1} m_{2}}{\left|\boldsymbol{\lambda}_{e}\right|^{3}}\left(1+\frac{1}{M_{2}}\left(\sum_{i=0}^{k} \frac{\alpha_{i}}{\left|\boldsymbol{\lambda}^{e}\right|^{2 i}}\left(\frac{1}{(1+\rho)^{2 i+2}}-\frac{1}{\rho^{2 i+2}}\right)\right)\right) \\
\widetilde{T}_{2}(\rho)=\sum_{i=0}^{k} \frac{G \alpha_{i}}{\left|\boldsymbol{\lambda}^{e}\right|^{2 i+3}}\left(\frac{m_{1}}{(1+\rho)^{2 i+2}}+\frac{m_{2}}{\rho^{2 i+2}}\right)
\end{gathered}
$$

and the modulus of the angular velocity of the gyrostat is

$$
\left|\Omega_{e}\right|^{2}=\frac{\widetilde{T}_{1}(\rho)}{g_{1}} .
$$

Remark 1 If a solution of relative equilibrium of Euler type exists, in an approximate dynamics of order $k$, fixed $\left|\boldsymbol{\lambda}_{e}\right|$, the equation (24) has positive real solutions. The number of real roots of the equation (24) will depend, obviously, of the numerous parameters that exist in our system. Similar results would be obtained for the other two cases.

\subsection{Sufficient condition of existence}

The following proposition indicates how to build solutions of the eq. (12).

Proposition 7 Fixed $\left|\boldsymbol{\lambda}^{e}\right|$, let $\rho$ be a solution of the equation

$$
g_{2}\left((1+\rho) m_{1}+\rho m_{2}\right) \widetilde{T}_{1}(\rho)=g_{1} M_{2} \widetilde{T}_{2}(\rho)
$$

where the functions $\widetilde{T}_{1}(\rho)$ and $\widetilde{T}_{2}(\rho)$ are given for the case a) as

$$
\begin{gathered}
\widetilde{T}_{1}(\rho)=\frac{G m_{1} m_{2}}{\left|\boldsymbol{\lambda}_{e}\right|^{3}}\left(1+\frac{1}{M_{2}}\left(\sum_{i=0}^{k} \frac{\alpha_{i}}{\left|\boldsymbol{\lambda}^{e}\right|^{2 i}}\left(\frac{1}{(1+\rho)^{2 i+2}}-\frac{1}{\rho^{2 i+2}}\right)\right)\right) \\
\widetilde{T}_{2}(\rho)=\sum_{i=0}^{k} \frac{G \alpha_{i}}{\left|\boldsymbol{\lambda}^{e}\right|^{2 i+3}}\left(\frac{m_{1}}{(1+\rho)^{2 i+2}}+\frac{m_{2}}{\rho^{2 i+2}}\right),
\end{gathered}
$$


then $\mathbf{z}_{e}=\left(\Pi_{e}, \boldsymbol{\lambda}^{e}, \mathbf{p}_{\boldsymbol{\lambda}}^{e}, \boldsymbol{\mu}^{e}, \mathbf{p}_{\mu}^{e}\right)$ given by

$$
\begin{array}{ll}
\lambda^{e}=\left(\lambda^{e}, 0,0\right) \quad, \quad \mathbf{p}_{\lambda}^{e}=\left(0, g_{1} \omega_{e} \lambda^{e}, 0\right) \\
\boldsymbol{\mu}^{e}=\left(\mu^{e}, 0,0\right) \quad, \quad \mathbf{p}_{\mu}^{e}=\left(0, g_{2} \omega_{e} \mu^{e}, 0\right) \\
\Omega_{e}=\left(0,0, \omega_{e}\right) \quad, \quad \Pi_{e}=\left(0,0, C \omega_{e}+l\right)
\end{array}
$$

where

$$
\begin{gathered}
\boldsymbol{\mu}^{e}=\frac{\left((1+\rho) m_{1}+\rho m_{2}\right)}{M_{2}} \boldsymbol{\lambda}^{e} \\
\omega_{e}^{2}=\frac{\widetilde{T}_{1}(\rho)}{g_{1}}
\end{gathered}
$$

is a solution of relative equilibrium of Euler type, in an approximate dynamics of order $k$, in the configuration $S_{0} S_{2} S_{1}$. The total angular momentum of the system is given by

$$
\mathbf{L}=\left(0,0, C \omega_{e}+l+g_{1} \omega_{e}^{2} \lambda^{e}+g_{2} \omega_{e}^{2} \mu^{e}\right)
$$

where $l$ is the gyrostatic momentum.

Let us see the existence and number of solutions for the approximate dynamics of order zero and one respectively. For superior order it is possible to use a similar technical.

\subsection{Eulerian relative equilibria in an approximate dy- namics of order zero}

For the configuration $S_{0} S_{2} S_{1}$, in an approximate dynamics of order zero, we have

$$
\begin{gathered}
\widetilde{T}_{1}(\rho)=\frac{G m_{1} m_{2}}{\left|\boldsymbol{\lambda}_{e}\right|^{3}}\left(1+\frac{m_{0}}{M_{2}}\left(\frac{1}{(1+\rho)^{2}}-\frac{1}{\rho^{2}}\right)\right) \\
\widetilde{T}_{2}(\rho)=\frac{G m_{0}}{\left|\boldsymbol{\lambda}_{e}\right|^{3}}\left(\frac{m_{1}}{(1+\rho)^{2}}+\frac{m_{2}}{\rho^{2}}\right)
\end{gathered}
$$

The equation (24) is equivalent to the following polynomial equation

$$
\begin{aligned}
& \left(m_{1}+m_{2}\right) \rho^{5}+\left(3 m_{1}+2 m_{2}\right) \rho^{4}+\left(3 m_{1}+m_{2}\right) \rho^{3}+ \\
& -\left(3 m_{0}+m_{2}\right) \rho^{2}-\left(3 m_{0}+2 m_{2}\right) \rho-\left(m_{0}+m_{2}\right)
\end{aligned}
$$


This equation has an unique positive real solution, then in this case for the approximate dynamics of order zero, there exist an unique relative equilibrium of Euler type.

On the other hand, one has

$$
\left|\Omega_{e}\right|^{2}=\frac{1}{g_{1}} \frac{G m_{1} m_{2}}{\left|\boldsymbol{\lambda}^{e}\right|^{3}}\left(1+\frac{m_{0}}{M_{2}}\left(\frac{1}{(1+\rho)^{2}}-\frac{1}{\rho^{2}}\right)\right)
$$

being $\rho$ the only one positive solution of the equation (34).

The following proposition gathers the results about relative equilibria of Euler type in an approximate dynamics of order zero in any of the cases previously mentioned $a$ ), $b$ ) or $c$ ).

Proposition 8 1. If $\rho$ is the unique positive root of the equation

$$
\begin{aligned}
& \left(m_{1}+m_{2}\right) \rho^{5}+\left(3 m_{1}+2 m_{2}\right) \rho^{4}+\left(3 m_{1}+m_{2}\right) \rho^{3}+ \\
& -\left(3 m_{0}+m_{2}\right) \rho^{2}-\left(3 m_{0}+2 m_{2}\right) \rho-\left(m_{0}+m_{2}\right)=0
\end{aligned}
$$

with

$$
\left|\Omega_{e}\right|^{2}=\frac{1}{g_{1}} \frac{G m_{1} m_{2}}{\left|\boldsymbol{\lambda}_{e}\right|^{3}}\left(1+\frac{m_{0}}{M_{2}}\left(\frac{1}{(1+\rho)^{2}}-\frac{1}{\rho^{2}}\right)\right)
$$

then $\mathbf{z}_{e}=\left(\Pi_{e}, \boldsymbol{\lambda}^{e}, \mathbf{p}_{\boldsymbol{\lambda}}^{e}, \boldsymbol{\mu}^{e}, \mathbf{p}_{\mu}^{e}\right)$, given by

$$
\begin{array}{ccc}
\boldsymbol{\lambda}^{e}=\left(\lambda^{e}, 0,0\right) & \boldsymbol{\mu}^{e}=\left(\mu^{e}, 0,0\right) & \Omega_{e}=\left(0,0, \omega_{e}\right) \\
\mathbf{p}_{\boldsymbol{\lambda}}^{e}=\left(0, g_{1} \omega_{e} \lambda^{e}, 0\right) & \mathbf{p}_{\boldsymbol{\mu}}^{e}=\left(0, g_{2} \omega_{e} \mu^{e}, 0\right) & \Pi_{e}=\left(0,0, C \omega_{e}+l\right)
\end{array}
$$

is the unique solution of relative equilibrium of Euler type in the configuration $S_{0} S_{2} S_{1}$.

2. If $\rho \in(-1,0)$ is the unique root of the equation

$$
\begin{gathered}
\left(m_{1}+m_{2}\right) \rho^{5}+\left(3 m_{1}+2 m_{2}\right) \rho^{4}+\left(3 m_{1}+m_{2}\right) \rho^{3}+ \\
+\left(3 m_{0}+2 m_{1}+m_{2}\right) \rho^{2}+\left(3 m_{0}+2 m_{2}\right) \rho+\left(m_{0}+m_{2}\right)=0
\end{gathered}
$$

with

$$
\left|\Omega_{e}\right|^{2}=\frac{1}{g_{1}} \frac{G m_{1} m_{2}}{\left|\boldsymbol{\lambda}_{e}\right|^{3}}\left(1+\frac{m_{0}}{M_{2}}\left(\frac{1}{\rho^{2}}-\frac{1}{(1+\rho)^{2}}\right)\right) .
$$


then $\mathbf{z}_{e}=\left(\Pi_{e}, \boldsymbol{\lambda}^{e}, \mathbf{p}_{\boldsymbol{\lambda}}^{e}, \boldsymbol{\mu}^{e}, \mathbf{p}_{\mu}^{e}\right)$ given by

$$
\begin{array}{ll}
\lambda^{e}=\left(\lambda^{e}, 0,0\right) \quad, \quad \mathbf{p}_{\lambda}^{e}=\left(0, g_{1} \omega_{e} \lambda^{e}, 0\right) \\
\boldsymbol{\mu}^{e}=\left(\mu^{e}, 0,0\right) \quad, \quad \mathbf{p}_{\mu}^{e}=\left(0, g_{2} \omega_{e} \mu^{e}, 0\right) \\
\Omega_{e}=\left(0,0, \omega_{e}\right) \quad, \quad \Pi_{e}=\left(0,0, C \omega_{e}+l\right)
\end{array}
$$

is the unique solution of relative equilibrium of Euler type in the configuration $S_{2} S_{0} S_{1}$.

3. If $\rho \in(-\infty,-1)$ is the unique root of the equation

$$
\begin{gathered}
\left(m_{1}+m_{2}\right) \rho^{5}+\left(3 m_{1}+2 m_{2}\right) \rho^{4}+\left(2 m_{0}+3 m_{1}+m_{2}\right) \rho^{3}+ \\
+\left(3 m_{0}+m_{2}\right) \rho^{2}+\left(3 m_{0}+2 m_{2}\right) \rho+\left(m_{0}+m_{2}\right)=0
\end{gathered}
$$

with

$$
\left|\Omega_{e}\right|^{2}=\frac{1}{g_{1}} \frac{G m_{1} m_{2}}{\left|\boldsymbol{\lambda}_{e}\right|^{3}}\left(1+\frac{m_{0}}{M_{2}}\left(\frac{1}{\rho^{2}}+\frac{1}{(1+\rho)^{2}}\right)\right)
$$

then $\mathbf{z}_{e}=\left(\Pi_{e}, \boldsymbol{\lambda}^{e}, \mathbf{p}_{\boldsymbol{\lambda}}^{e}, \boldsymbol{\mu}^{e}, \mathbf{p}_{\mu}^{e}\right)$ given by

$$
\begin{array}{ll}
\boldsymbol{\lambda}^{e}=\left(\lambda^{e}, 0,0\right) \quad, \quad \mathbf{p}_{\boldsymbol{\lambda}}^{e}=\left(0, g_{1} \omega_{e} \lambda^{e}, 0\right) \\
\boldsymbol{\mu}^{e}=\left(\mu^{e}, 0,0\right) \quad, \quad \mathbf{p}_{\boldsymbol{\mu}}^{e}=\left(0, g_{2} \omega_{e} \mu^{e}, 0\right) \\
\Omega_{e}=\left(0,0, \omega_{e}\right) \quad, \quad \Pi_{e}=\left(0,0, C \omega_{e}+l\right)
\end{array}
$$

is the unique solution of relative equilibrium of Euler type in the configuration $S_{2} S_{1} S_{0}$.

\subsection{Eulerian relative equilibria in an approximate dy- namics of order one}

For the approximate dynamics of order one, after carrying out the appropriate calculations, the equation (24) corresponding to the configuration $S_{0} S_{2} S_{1}$, is reduced to the study of the positive real roots of the equation 


$$
\begin{gathered}
m_{0} a^{2}\left(m_{1}+m_{2}\right) \rho^{9}+m_{0} a^{2}\left(5 m_{1}+4 m_{2}\right) \rho^{8}+m_{0} a^{2}\left(10 m_{1}+6 m_{2}\right) \rho^{7}+ \\
3 m_{0} a^{2}\left(3 m_{1}+m_{2}-m_{0}\right) \rho^{6}+3 m_{0} a^{2}\left(m_{1}-m_{2}-3 m_{0}\right) \rho^{5}-\left(6 m_{0} m_{2} a^{2}+\right. \\
\left.+10 m_{0}^{2} a^{2}+\alpha_{1}\left(m_{1}+m_{2}+5 m_{0}\right)\right) \rho^{4}-\left(4 m_{0} m_{2} a^{2}+5 m_{0}^{2} a^{2}+\right. \\
\left.+\alpha_{1}\left(10 m_{0}+4 m_{2}\right)\right) \rho^{3}-\left(m_{0} m_{2} a^{2}+m_{0}^{2} a^{2}+\alpha_{1}\left(6 m_{2}+10 m_{0}\right)\right) \rho^{2}- \\
-\alpha_{1}\left(5 m_{0}+4 m_{2}\right) \rho-\alpha_{1}\left(m_{0}+m_{2}\right)=0
\end{gathered}
$$

where $a=\left|\boldsymbol{\lambda}_{e}\right|$ and $\alpha_{1}=\frac{3 m_{0}(C-A)}{2}$, being $C$ and $A$ the principal moments of inertia of the gyrostat.

To study the positive real roots of this equation, after a detailed analysis of the same one, it can be expressed in the following way

$$
\beta_{1}=R_{1}(\rho)=\frac{m_{0} a^{2} \rho^{2}(\rho+1)^{2} p_{0}(\rho)}{q_{0}(\rho)}
$$

being $\beta_{1}=(3 / 2)(C-A), p_{0}$ the polynomial of grade five that determines the relative equilibria in the approximate dynamics of order 0 , that is given by the formula (34), and the polynomial $q_{0}$ comes determined by the following expression

$$
\begin{aligned}
& q_{0}(\rho)=\left(m_{1}+m_{2}+5 m_{0}\right) \rho^{4}+\left(4 m_{2}+10 m_{0}\right) \rho^{3}+ \\
& +\left(6 m_{2}+10 m_{0}\right) \rho^{2}+\left(4 m_{2}+5 m_{0}\right) \rho+\left(m_{0}+m_{2}\right)
\end{aligned}
$$

The rational function $R_{1}(\rho)$, for any value of $m_{0}, m_{1}, m_{2}$, always presents a minimum $\xi_{1}$ located among 0 and $\rho_{0}$, being this last value the only one positive zero of the polynomial $p_{0}(\rho)$.

By virtue of these statements the following result is obtained.

Proposition 9 In the approximate dynamics of order one, if the gyrostat $S_{0}$ is prolate $\left(\beta_{1}<0\right)$, we have:

1. $\beta_{1}<R_{1}\left(\xi_{1}\right)$, then relative equilibria of Euler type don't exist.

2. $\beta_{1}=R_{1}\left(\xi_{1}\right)$, then there exists an unique 1-parametric family of relative equilibria of Euler type.

3. $R_{1}\left(\xi_{1}\right)<\beta_{1}<0$, then two 1-parametric families of relative equilibria of Euler type exist. 


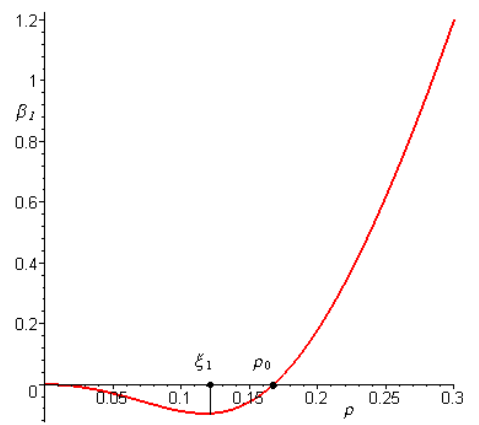

Figure 3: Function $R_{1}(\rho)$

If $S_{0}$ is oblate $\left(\beta_{1}>0\right)$, then there exists an unique 1-parametric family of relative equilibria of Euler type.

Similarly for the configuration $S_{2} S_{0} S_{1}$ we obtain the following result.

Proposition 10 In the approximate dynamics of order one, if $m_{1} \neq m_{2}$ and the gyrostat $S_{0}$ is oblate, then there exists an unique 1-parametric family of relative equilibria of Euler type; on the other hand, if the gyrostat $S_{0}$ is prolate and we have:

1. $\beta_{1}<R_{1}\left(\xi_{1}\right)$, then there exists an unique 1-parametric family of relative equilibria of Euler type.

2. $\beta_{1}=R_{1}\left(\xi_{1}\right)$, then two 1-parametric families of relative equilibria of Euler type exist.

3. $R_{1}\left(\xi_{1}\right)<\beta_{1}<0$, then three 1-parametric families of relative equilibria of Euler type exist. If $m_{1}=m_{2}$ and $S_{0}$ is oblate, then relative equilibria of Euler type don't exist; but if $S_{0}$ is prolate we have:

4. $R_{1}(-1 / 2)<\beta_{1}<0$, then two 1-parametric families of relative equilibria of Euler type exist.

5. $\beta_{1}=R_{1}(-1 / 2)$, there exists an unique 1-parametric family of relative equilibria of Euler type.

6. $\beta_{1}<R_{1}(-1 / 2)$, then relative equilibria of Euler type don't exist. 


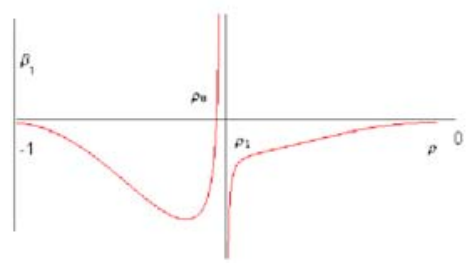

Figure 4: Function $R_{1}(\rho)$ for $m_{1} \neq m_{2}$

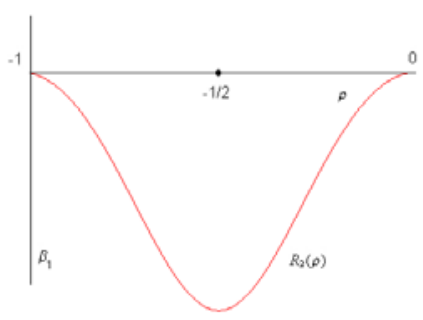

Figure 5: Function $R_{1}(\rho)$ for $m_{1}=m_{2}$

\section{Relative equilibria of Lagrange type}

\subsection{Necessary condition of existence}

If $\mathbf{z}_{e}=\left(\Pi_{e}, \boldsymbol{\lambda}^{e}, \mathbf{p}_{\boldsymbol{\lambda}}^{e}, \boldsymbol{\mu}^{e}, \mathbf{p}_{\mu}^{e}\right)$ is a relative equilibrium of Lagrange type, in an approximate dynamics of order $k$, the following identities are verified

$$
\begin{aligned}
& \boldsymbol{\lambda}^{e} \times\left(\nabla_{\boldsymbol{\lambda}} \mathcal{V}^{(k)}\right)_{e}=\mathbf{0}, \quad g_{1}\left|\Omega_{e}\right|^{2}\left(\boldsymbol{\lambda}^{e} \times \boldsymbol{\mu}^{e}\right)=\left(\boldsymbol{\nabla}_{\boldsymbol{\lambda}} \mathcal{V}^{(k)}\right)_{e} \times \boldsymbol{\mu}^{e} \\
& \boldsymbol{\mu}^{e} \times\left(\boldsymbol{\nabla}_{\boldsymbol{\mu}} \mathcal{V}^{(k)}\right)_{e}=\mathbf{0}, \quad g_{2}\left|\Omega_{e}\right|^{2}\left(\boldsymbol{\lambda}^{e} \times \boldsymbol{\mu}^{e}\right)=\boldsymbol{\lambda}^{e} \times\left(\boldsymbol{\nabla}_{\boldsymbol{\mu}} \mathcal{V}^{(k)}\right)_{e}
\end{aligned}
$$

in the relative equilibria, from the equation (5) we deduce

$$
\begin{aligned}
& \left(\widetilde{A}_{12}\right)_{e}\left(\boldsymbol{\lambda}^{e} \times \boldsymbol{\mu}^{e}\right)=\mathbf{0}, \quad g_{1}\left|\Omega_{e}\right|^{2}\left(\boldsymbol{\lambda}^{e} \times \boldsymbol{\mu}^{e}\right)=\left(\widetilde{A}_{11}\right)_{e}\left(\boldsymbol{\lambda}^{e} \times \boldsymbol{\mu}^{e}\right) \\
& \left(\widetilde{A}_{21}\right)_{e}\left(\boldsymbol{\lambda}^{e} \times \boldsymbol{\mu}^{e}\right)=\mathbf{0}, \quad g_{2}\left|\Omega_{e}\right|^{2}\left(\boldsymbol{\lambda}^{e} \times \boldsymbol{\mu}^{e}\right)=\left(\widetilde{A}_{22}\right)_{e}\left(\boldsymbol{\lambda}^{e} \times \boldsymbol{\mu}^{e}\right)
\end{aligned}
$$

being $\left(\widetilde{A}_{i j}\right)_{e}$ the evaluation in the equilibria of $\widetilde{A}_{i j}$.

Concluding, we have the following relations

$$
\left(\widetilde{A}_{12}\right)_{e}=\left(\widetilde{A}_{21}\right)_{e}=0, \quad\left|\Omega_{e}\right|^{2}=\frac{\left(\widetilde{A}_{11}\right)_{e}}{g_{1}}=\frac{\left(\widetilde{A}_{22}\right)_{e}}{g_{2}} .
$$

and the following property is obtained. 
Proposition 11 Let $\mathbf{z}_{e}=\left(\Pi_{e}, \boldsymbol{\lambda}^{e}, \mathbf{p}_{\boldsymbol{\lambda}}^{e}, \boldsymbol{\mu}^{e}, \mathbf{p}_{\mu}^{e}\right)$ be a relative equilibrium of Lagrange type. Then we have

$$
\begin{gathered}
\left(\widetilde{A}_{12}\right)_{e}=0 \\
g_{2}\left(\widetilde{A}_{11}\right)_{e}=g_{1}\left(\widetilde{A}_{22}\right)_{e}
\end{gathered}
$$

with

$$
\left|\Omega_{e}\right|^{2}=\frac{\left(\widetilde{A}_{11}\right)_{e}}{g_{1}}
$$

Using the expressions of $\widetilde{A}_{i j}$, given in (6), we prove the following result.

Proposition 12 If $\mathbf{z}_{e}=\left(\Pi_{e}, \boldsymbol{\lambda}^{e}, \mathbf{p}_{\boldsymbol{\lambda}}^{e}, \boldsymbol{\mu}^{e}, \mathbf{p}_{\mu}^{e}\right)$ is a relative equilibrium of Lagrange type in an approximate dynamics of order $k$, then denoting by $\left|\boldsymbol{\lambda}^{e}\right|=Z$, $\left|\boldsymbol{\mu}^{e}+\frac{m_{2}}{M_{2}} \boldsymbol{\lambda}^{e}\right|=X,\left|\boldsymbol{\mu}^{e}-\frac{m_{1}}{M_{2}} \boldsymbol{\lambda}^{e}\right|=Y$, the system of equations

$$
\left\{\begin{aligned}
X^{2 k+3} & =\sum_{i=0}^{k} \beta_{i} Z^{3} X^{2(k-i)} \\
Y^{2 k+3} & =\sum_{i=0}^{k} \beta_{i} Z^{3} Y^{2(k-i)}
\end{aligned}\right.
$$

has positive real solutions.

Remark 2 The parameters that have influence in the study of the number of the different configurations of relative equilibrium of Lagrange type will be $Z$ and $\beta_{i}(i=1,2, \ldots, k)$.

\subsection{Sufficient condition of existence}

If we fix $Z$ and there exist $X$ and $Y$ verifying the system of equations

$$
\left\{\begin{aligned}
X^{2 k+3} & =\sum_{i=0}^{k} \beta_{i} Z^{3} X^{2(k-i)} \\
Y^{2 k+3} & =\sum_{i=0}^{k} \beta_{i} Z^{3} Y^{2(k-i)}
\end{aligned}\right.
$$

with respect to an appropriate reference system, we can build relative equilibria of Lagrange type. If $X=Y \neq Z$ is a solution of the previous system, then 
the $S_{i}(\mathrm{i}=0,1,2)$ form an isosceles triangle. If $X \neq Y \neq Z$ then the $S_{i}$ form a scalene triangle.

The following proposition whose demonstration decreases to verify the equations of the equilibria, shows how are the equilibria of Lagrange type when $S_{0}, S_{1}, S_{2}$ forms an isosceles triangle. Using the same reasonings we could describe the equilibria of Lagrange type when $S_{0}, S_{1}, S_{2}$ form a scalene triangle.

Proposition 13 With respect to an appropriate reference system we have that $\mathbf{z}_{e}=\left(\Pi_{e}, \boldsymbol{\lambda}^{e}, \mathbf{p}_{\boldsymbol{\lambda}}^{e}, \boldsymbol{\mu}^{e}, \mathbf{p}_{\mu}^{e}\right)$ given by

$$
\begin{array}{ll}
\boldsymbol{\lambda}^{e}=\left(x_{1}, y_{1}, 0\right) & , \quad \mathbf{p}_{\boldsymbol{\lambda}}^{e}=g_{1} \omega_{e}\left(-y_{1}, x_{1}, 0\right) \\
\boldsymbol{\mu}^{e}=\left(x_{2}, y_{2}, 0\right) & , \quad \mathbf{p}_{\boldsymbol{\mu}}^{e}=g_{2} \omega_{e}\left(-y_{2}, x_{2}, 0\right) \\
\Omega_{e}=\left(0,0, \omega_{e}\right) \quad, \quad \Pi_{e}=\left(0,0, C \omega_{e}+l\right)
\end{array}
$$

with

$$
\begin{gathered}
x_{1}=X(1-\cos \theta) \quad, \quad y_{1}=-X \sin \theta \\
x_{2}=\frac{X\left(m_{1}+m_{2} \cos \theta\right)}{M_{2}}, \quad y_{2}=\frac{m_{2} X \sin \theta}{M_{2}} \\
\omega_{e}^{2}=G M_{1} \sum_{i=0}^{k} \frac{\beta_{i}}{X^{2 i+3}}\left(\beta_{0}=1\right)
\end{gathered}
$$

being $\theta$ the angle of the isosceles triangle $S_{0} S_{1} S_{2}$, are the relative equilibria of Lagrange type. The total angular momentum vector of the system is given by

$$
\mathbf{L}=\left(0,0, C \omega_{e}+l+\omega_{e}^{2} \sum_{i=1}^{2} g_{i}\left(x_{i}^{2}+y_{i}^{2}\right)\right)
$$

Next we study the relative equilibria of Lagrange type in the approximate dynamics of order zero and one.

\subsection{Relative equilibria of Lagrange type in an approxi- mate dynamics of order zero}

When $k=0$, the equations (53) are

$$
\left\{\begin{array}{l}
X^{3}=Z^{3} \\
Y^{3}=Z^{3}
\end{array}\right.
$$


then easily we deduce that $X=Y=Z$. That is to say $S_{0}, S_{1}$ and $S_{2}$ form an equilateral triangle. Also, we obtain

$$
\left|\Omega_{e}\right|^{2}=\frac{G M_{1}}{Z^{3}}
$$

On the other hand one parametrization of these equilibria $\mathbf{z}_{e}=\left(\Pi_{e}, \boldsymbol{\lambda}^{e}, \mathbf{p}_{\boldsymbol{\lambda}}^{e}, \boldsymbol{\mu}^{e}, \mathbf{p}_{\mu}^{e}\right)$ is given by

$$
\begin{array}{ll}
\boldsymbol{\lambda}^{e}=\left(x_{1}, y_{1}, 0\right), & \mathbf{p}_{\boldsymbol{\lambda}}^{e}=g_{1} \omega_{e}\left(-y_{1}, x_{1}, 0\right) \\
\boldsymbol{\mu}^{e}=\left(x_{2}, y_{2}, 0\right), & \mathbf{p}_{\boldsymbol{\mu}}^{e}=g_{2} \omega_{e}\left(-y_{2}, x_{2}, 0\right) \\
\Omega_{e}=\left(0,0, \omega_{e}\right), & \Pi_{e}=\left(0,0, C \omega_{e}+l\right)
\end{array}
$$

being

$$
\begin{gathered}
x_{1}=\frac{Z}{2}, \quad y_{1}=-\frac{\sqrt{3} Z}{2} \\
x_{2}=\frac{Z\left(m_{2}+2 m_{1}\right)}{2 M_{2}}, \quad y_{2}=\frac{m_{2} \sqrt{3} Z}{2 M_{2}} \\
\omega_{e}^{2}=\frac{G M_{1}}{Z^{3}}
\end{gathered}
$$

This parametrization of the relative equilibria will be of great utility for the posterior study of the stability of the same ones.

\subsection{Relative equilibria of Lagrange type in an approxi- mate dynamics of order one}

For $k=1$, the equations (53) are

$$
\left\{\begin{array}{l}
X^{5}-Z^{3} X^{2}-\beta_{1} Z^{3}=0 \\
Y^{5}-Z^{3} Y^{2}-\beta_{1} Z^{3}=0
\end{array}\right.
$$

being $Z$ and $\beta_{1}$ parameters. Let us proceed next to study the number of positive real roots of the polynomial

$$
p(X)=X^{5}-Z^{3} X^{2}-\beta_{1} Z^{3}
$$

according to the values of the parameters $Z$ and $\beta_{1}$.

Applying the rule of the signs of Descartes, if $\beta_{1} \geq 0$ then this polynomial can only have a positive real root. 
If $\beta_{1}<0$ then we can have two positive real roots, a real root (positive) or none. The discriminant of the polynomial, denoted by $\operatorname{discrim}(p, X)$, comes given by

$$
\operatorname{discrim}(p, X)=\beta_{1} Z^{12}\left(3125 \beta_{1}^{3}+108 Z^{6}\right) .
$$

Then if $\operatorname{discrim}(p, X)<0$, the polynomial $p$ has two real roots, if $\operatorname{discrim}(p, x)=$ 0 , it has a positive double root, while if $\operatorname{discrim}(p, x)>0$, it has not positive root.

The discriminant is zero when the following relation is verified

$$
\beta_{1}=-\frac{3 \sqrt[3]{20}}{25} Z^{2}
$$

By the previous results we can make a complete study of the bifurcations of the equilibria in an approximate dynamics of order 1.

Proposition 14 Let $\mathbf{z}_{e}=\left(\Pi_{e}, \boldsymbol{\lambda}^{e}, \mathbf{p}_{\boldsymbol{\lambda}}^{e}, \boldsymbol{\mu}^{e}, \mathbf{p}_{\mu}^{e}\right)$ be a relative equilibrium of Lagrange type, in an approximate dynamics of order one. Then:

1. If $\beta_{1} \geq 0$ (gyrostat oblate) an only 2-parametric family exists forming $S_{0}, S_{1}, S_{2}$ an isosceles triangle.

2. If $\beta_{1}<0$ (gyrostat prolate) then

a1) If $\frac{-7 Z^{2}}{32}<\beta_{1}<0$, there are two types of relative equilibria:

- One 2-parametric family of relative equilibria forming $S_{0}, S_{1}$, $S_{2}$ an isosceles triangle with $X=Y \neq Z$.

- Two 2-parametric families of relative equilibria forming $S_{0}, S_{1}$, $S_{2}$ a scalene triangle with $X \neq Y \neq Z$.

a2) If $-\frac{3 \sqrt[3]{20}}{25} Z^{2}<\beta_{1}<\frac{-7 Z^{2}}{32}$, there are two types of relative equilibria:

- Two 2-parametric families of relative equilibria forming $S_{0}, S_{1}$, $S_{2}$ an isosceles triangle with $X=Y \neq Z$.

- Four 2-parametric families of relative equilibria forming $S_{0}, S_{1}$, $S_{2}$ an scalene triangle with $X \neq Y \neq Z$.

b) If $\beta_{1}=-\frac{3 \sqrt[3]{20}}{25} Z^{2}$ an only 2-parametric family exists forming $S_{0}, S_{1}$, $S_{2}$ an isosceles triangle, with $X=Y \neq Z$.

c) If $\beta_{1}<-\frac{3 \sqrt[3]{20}}{25} Z^{2}$ relative equilibria don't exist. 


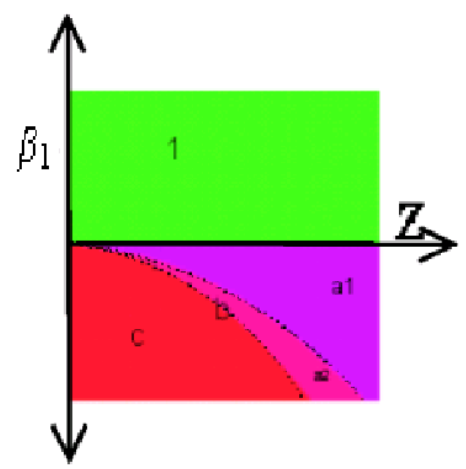

Figure 6: Bifurcations of the equilibria in the plane $\beta_{1} Z$

On the other hand we have that $\mathbf{z}_{e}=\left(\Pi_{e}, \boldsymbol{\lambda}^{e}, \mathbf{p}_{\boldsymbol{\lambda}}^{e}, \boldsymbol{\mu}^{e}, \mathbf{p}_{\mu}^{e}\right)$ given by

$$
\begin{array}{ll}
\boldsymbol{\lambda}^{e}=\left(x_{1}, y_{1}, 0\right), & \mathbf{p}_{\boldsymbol{\lambda}}^{e}=g_{1} \omega_{e}\left(-y_{1}, x_{1}, 0\right) \\
\boldsymbol{\mu}^{e}=\left(x_{2}, y_{2}, 0\right), & \mathbf{p}_{\boldsymbol{\mu}}^{e}=g_{2} \omega_{e}\left(-y_{2}, x_{2}, 0\right) \\
\Omega_{e}=\left(0,0, \omega_{e}\right), & \Pi_{e}=\left(0,0, C \omega_{e}+l\right)
\end{array}
$$

with

$$
\begin{gathered}
\omega_{e}^{2}=G M_{1}\left(\frac{1}{X^{3}}+\frac{\beta_{1}}{X^{5}}\right) \\
x_{1}=X(1-\cos \theta), \quad y_{1}=-X \sin \theta \\
x_{2}=\frac{X\left(m_{1}+m_{2} \cos \theta\right)}{M_{2}}, \quad y_{2}=\frac{m_{2} X \sin \theta}{M_{2}}
\end{gathered}
$$

being

$$
\cos \theta=\frac{2 X^{2}-Z^{2}}{2 X^{2}}, \quad \sin \theta=\frac{Z \sqrt{4 X^{2}-Z^{2}}}{2 X^{2}}
$$

describes a parametrization of the equilibria of Lagrange type, in an approximate dynamics of order one, when $S_{0}, S_{1}, S_{2}$ form an isosceles triangle. The total angular momentum of the system is given by

$$
\mathbf{L}=\left(0,0, C \omega_{e}+l+\omega_{e}^{2} \sum_{i=1}^{2} g_{i}\left(x_{i}^{2}+y_{i}^{2}\right)\right)
$$


Remark 3 It is easy to see that when the gyrostat is oblate, in the previous equilibria, it rotates quicker around the principal axis of inertia $C$ that when the gyrostat is prolate.

In a same way the equilibria type Lagrange could be described in an approximate dynamics of order one when $S_{0}, S_{1}, S_{2}$ forms a scalene triangle

Remark 4 To study the relative equilibria of Lagrange type, in an approximate dynamics of order $k$ anyone, we should study the positive real solutions of the equation

$$
X^{2 k+3}-\sum_{i=0}^{k} \beta_{i} Z^{3} X^{2(k-i)}=0
$$

If we know the number of positive roots in the approximate dynamics of order $k$, we can know the number of positive roots of the polynomial equation that arises in the approximate dynamics of order $k+1$. This study reduces to calculate the number of positive roots of the equation

$$
\beta_{k+1}=\frac{X^{2}\left[X^{2 k+3}-\sum_{i=0}^{k} \beta_{i} Z^{3} X^{2(k-i)}\right]}{Z^{3}}
$$

\section{Conclusions and future works}

- The approximate dynamics of a gyrostat (or rigid body) in Newtonian interaction with two spherical or punctual rigid bodies is considered.

- For order zero and one a complete study of equilibria of Euler and Lagrange type is made.

- Diverse results, which had been obtained by means of classic methods in previous works, have been obtained and generalized in a different way. And other results, not previously considered, have been studied.

- The bifurcations of some Eulerian and Lagrangian relative equilibria are given.

- Numerous problems are open, and among them it is necessary to consider the study of the "inclined" relative equilibria, in which $\Omega_{e}$ form an angle $\alpha \neq 0$ and $\pi / 2$ with the vector $\boldsymbol{\lambda}^{e} \times \boldsymbol{\mu}^{e}$ and the stability of the relative equilibria obtained here.

\section{Acknowledgements}


This research was partially supported by the Spanish Ministerio de Ciencia y Tecnología (Project BFM2003-02137) and by the Consejería de Educación y Cultura de la Comunidad Autónoma de la Región de Murcia (Project Séneca 2002: PC-MC/3/00074/FS/02).

\section{References}

[1] G. N. Duboshin, The problem of three rigid bodies, Celest. Mech.\& Dyn. Astron., 33 (1984), 31-47.

[2] C. Fanny, E. Badaoui, Relative equilibrium in the three-body problem with a rigid body, Celest. Mech. 6 Dyn. Astron., 69 (1997), 293-315.

[3] A. Maciejewski, Reduction, relative equilibria and potential in the two rigid bodies problem, Celest. Mech.\& Dyn. Astron., 63 (1995), 1-28.

[4] F. Mondéjar, A. Vigueras, The Hamiltonian dynamics of the two gyrostats problem, Celest. Mech.\& Dyn. Astron., 73 (1999), 303-312.

[5] F. Mondéjar, A. Vigueras, S. Ferrer, Symmetries, reduction and relative equilibria for a gyrostat in the three-body problem, Celest. Mech.\& Dyn. Astron., 81 (2001), 45-50.

[6] J. A. Vera, Reducciones, equilibrios y estabilidad en dinámica de sólidos rígidos y giróstatos, $\mathrm{PhD}$ Dissertation, Universidad Politécnica de Cartagena, Spain. 2004.

[7] J. A. Vera, A. Vigueras, Reduction, relative equilibria and stability for a gyrostat in the n-body problem, In: M.C. López de Silanes et al. (eds.) Monografías del Seminario Matemático García de Galdeano (VIII Journées Zaragoza-Pau de Mathematiques Appliquées et de Statistiques), vol. 31, 257-271, Servicio de Publicaciones de la Universidad de Zaragoza, Zaragoza, Spain. 2004.

[8] J. A. Vera, A. Vigueras, Hamiltonian dynamics of a gyrostat in the nbody problem: relative equilibria, Celest. Mech.\& Dyn. Astron., 94(3) (2006), 289-315.

[9] V. V. Vidiakin, Euler solutions in the problem of translational-rotational motion of three-rigid bodies, Celest. Mech.\& Dyn. Astron. 16 (1977), 509526.

[10] L. S. Wang, P. S. Krishnaprasad, J. H. Maddocks, Hamiltonian dynamics of a rigid body in a central gravitational field, Celest. Mech. $\&$ Dyn. Astron. 50 (1991), 349-386. 
[11] L. S. Wang, K. Y. Lian, P. T. Chen, Steady motions of gyrostat satellites and their stability, IEEE Transactions on Automatic Control 40(10) (1995), 1732-1743.

[12] S. G. Zhuravlev, A. A. Petrutskii, Current state of the problem of translational-rotational motion of three-rigid bodies, Soviet Astron. 34 (1990), 299-304.

Received: June 13, 2006 